\title{
El hueso, la obesidad y su interacción endocrina
}

\author{
María Camila Romero Ortiz', Jhoan Sebastián Roncancio Muñoz $z^{2}$, Mónica Alejandra Bernal ${ }^{1}$, \\ Laura Alexandra Martínez Jiménez $z^{3}$, Julián David Rincón Lozano 3 , Juan Carlos Pulido Urbano, \\ David Camilo Jaramillo Castillo ${ }^{3}$, Roberto Franco Vega ${ }^{4}$, Luis Miguel Maldonado Acosta ${ }^{4}$, Juan \\ Manuel Arteaga Díaz ${ }^{4}$, Jorge Eduardo Caminos ${ }^{5}$, Juan Jose Rincón Ramírez ${ }^{3}$
}

${ }^{1}$ Residentes de Endocrinología, Departamento de Medicina Interna, Universidad Nacional de Colombia

${ }^{2}$ Residente de Medicina Interna, Departamento de Medicina Interna, Universidad Nacional de Colombia

${ }^{3}$ Estudiantes de pregrado Medicina, Departamento de Medicina Interna, Universidad Nacional de Colombia

${ }^{4}$ Profesor Titular de Posgrado, Endocrinología, Departamento de Medicina Interna, Universidad Nacional de Colombia

${ }^{5}$ Profesor Titular, director Unidad de Bioquímica, Departamento de Fisiología y Bioquímica.

Correspondencia: María Camila Romero Ortiz

Correo electrónico: mcromeroo@unal.edu.co

Conflicto de interés. Ninguno de los autores declara tener conflictos de interés

Fecha de recepción: 6/09/2017

Fecha de aceptación: 10/10/2017

\section{Resumen}

$\mathrm{L}$ a asociación entre la obesidad y la densidad mineral ósea ha sido un punto controversial al momento de establecer si existe una asociación positiva o negativa entre las mismas. Diversos estudios han propuesto que la obesidad es un factor protector del hueso, debido a la tensión mecánica dada por el peso corporal en la remodelación ósea. Otros estudios plantean que la relación es mucho más compleja debido a que el tejido adiposo y los osteoblastos provienen de líneas germinales comunes. Además, el adipocito tiene la capacidad de secretar diversas moléculas, entre ellas las adipocinas. Adicionalmente, el tejido adiposo es una de las principales fuentes de aromatasa, esto lo involucra en la conversión de andrógenos a estrógenos, que juegan un papel importante en el mantenimiento de la homeostasis ósea. Por lo tanto, se ha planteado el hueso como órgano blanco de diversas vías endocrinas y, a su vez, se considera un órgano endocrino que puede afectar otros órganos cuando está alterado. Por otra parte, se ha visto que la resistencia a la insulina en el contexto de la obesidad está asociada con inflamación crónica de bajo grado, deterioro funcional de órganos y alteración del metabolismo energético, que impacta la remodelación ósea.

\section{Abstract}

There is controversy over the effect of obesity in bone mineral density. Several studies have proposed that obesity is a protective factor of the bone by the mechanical tension that favors the bone remodeling. However, other studies suggest that this relationship is more complex because both tissues come from a common germ line; emphasizing that the adipocyte secretes diverse molecules, among them adipocinas. In addition, adipose tissue has aromatases that convert androgens into estrogens, having an importance in bone homeostasis. Therefore, the bone has been raised as a target organ of various endocrine pathways, which may affect other organs when it is altered. On the other hand, it has been shown that insulin resistance in the context of obesity is associated with chronic low-grade inflammation, functional impairment of organs and impaired energy metabolism, which impacts bone remodeling.

\section{Palabras clave}

Obesidad, Densidad ósea, Insulina, Factor de crecimiento similar a la insulina, Vitamina D, Leptina, Adiponectina, Estradiol, Testosterona.

\section{Keywords}

Obesity, Bone density, Insulin, Insulin-like growth factor, Vitamin D, Leptin, Adiponectin, Estradiol, Testosterone.

\section{Introducción}

Actualmente, la obesidad se ha convertido en una epidemia creciente a nivel mundial, incidiendo en la aparición de múltiples enfermedades de alto costo e impacto en morbilidad y mortalidad. Según la Organización Mundial de la Salud (OMS) la obesidad se define como una acumulación anormal o excesiva de grasa que constituye un riesgo para la salud, la cual es reconocida como un problema de salud pública a nivel mundial, dado su asociación con enfermedades crónicas no transmisibles tales como diabetes mellitus tipo 2, hipertensión arterial, síndrome de apnea e hipoapnea obstructiva del sueño y algunos tipos de cánceres, entre otros ${ }^{(1,2)}$.

En Colombia, la obesidad se ha determinado bajo los lineamientos de la OMS, definiéndola con base en un índice de masa corporal (IMC) mayor o igual a $30 \mathrm{~kg} / \mathrm{m}^{2}$. Actualmente, 
los estilos de vida sedentarios y dietas ricas en calorías han conllevado al incremento en la aparición de la obesidad en las personas entre los 18 y los 64 años, evidenciando mayor crecimiento en edades tempranas ${ }^{(3)}$. Se estima que la prevalencia para el 2015 es de 13,68\% para hombres y 21,05\% para mujeres. En países como Estados Unidos se estima que para el 2020 la prevalencia podría alcanzar el $75 \%$ de la población total ${ }^{(4)}$.

Entre las recientes asociaciones vinculadas a la obesidad se encuentra su papel frente a la densidad mineral ósea (DMO), determinada por múltiples factores y células que se encargan de mantener el adecuado equilibrio entre la formación de matriz y la resorción ósea ${ }^{(5)}$.

Entre los diferentes grupos celulares se encuentran las células madre mesenquimales pluripotenciales, que se pueden diferenciar a diversos tipos celulares, tales como los condrocitos, adipocitos y osteoblastos; estos últimos secretan colágeno tipo 1, osteopontina, osteocalcina y sialoproteína, que son constituyentes del tejido óseo y los principales encargados de promover el proceso de formación ósea ${ }^{(6)}$.

En contraparte se encuentran los osteoclastos, son células provenientes del linaje monocito-macrófago en la médula ósea. Dichas células tienen un papel fundamental en la resorción de la matriz mineral ósea, dado que promueven el recambio óseo y, a través de sus receptores hormonales, participan en la homeostasis del calcio. Entre las vías de interacción de los principales tipos celulares se encuentra la vía del receptor activador para el factor nuclear $\kappa B$ (RANK-RANKL) epicentro de acción de múltiples factores y hormonas ${ }^{(7,8)}$.
Como se mencionó previamente, existe una relación entre la obesidad y la DMO que actualmente es motivo de discusión, puesto que algunos autores afirman que el incremento de DMO está asociado con la obesidad, dado el mayor efecto mecánico que ejerce el peso sobre el hueso. Sin embargo, el incremento de la DMO también ha sido vinculado en áreas que no están expuestas a dicha tensión mecánica. En contraparte, estudios recientes que evalúan la DMO por medio de imágenes que proporcionan mayor resolución, demuestran su correlación negativa de la obesidad frente a la microestructura ósea ${ }^{(9)}$.

Debido a las actuales controversias que se han planteado sobre la relación entre la obesidad y la DMO, consideramos pertinente realizar una descripción de los diversos puntos de vista, enfatizando en la relación endocrina y los determinantes bioquímicos que inciden en la DMO (figura 1).

\section{Insulina}

La insulina es una hormona peptídica secretada por las células $\beta$ de los islotes pancreáticos de Langerhans en respuesta a elevación de la concentración de glucosa en la sangre. La función principal de la insulina es mantener los niveles de glucosa dentro de los rangos fisiológicos normales, mediante la regulación del metabolismo energético intermediario de lípidos y carbohidratos; por otra parte, estimula vías mitogénicas en múltiples grupos celulares ${ }^{(10)}$.

Dentro de las múltiples acciones de la insulina, se han descrito efectos directos e indirectos sobre el hueso mediante receptores en las células formadoras de hueso (osteoblastos) y

Figura 1. Interacciones endocrinas en el hueso. Efectos netos positivos y negativos de factores endocrinos sobre la DMO e interacción sobre formación y resorción ósea

\section{Insulina \\ Testosterona \\ Estradiol \\ Vitamina D \\ IGF-1 \\ Osteocalcina}

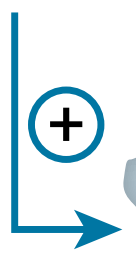

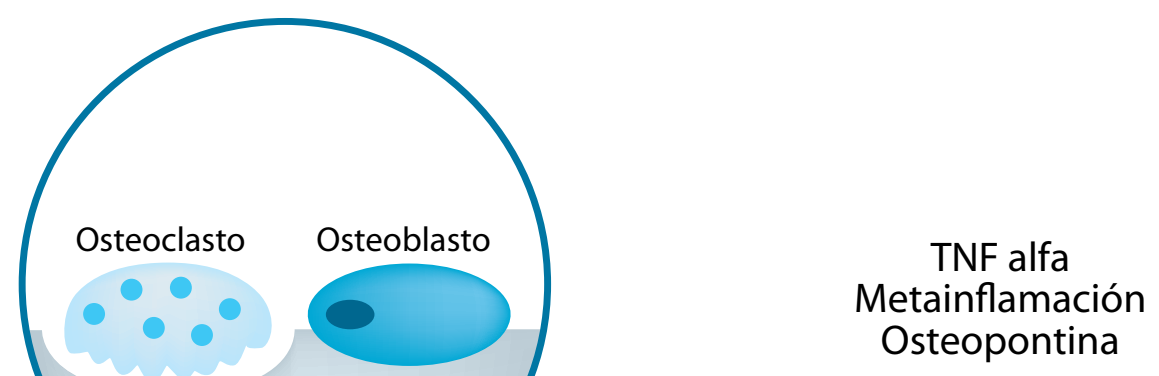

Osteopontina
Leptina
Adiponectina

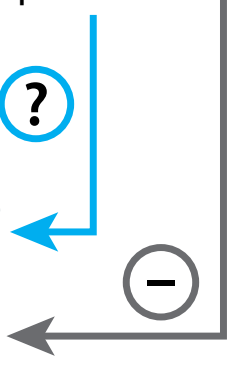


las células de resorción ósea (osteoclastos). Los efectos directos de la insulina parten de la acción en osteoblastos al promover su supervivencia por medio del aumento de la expresión de la osteocalcina. El osteoblasto secreta osteocalcina en su forma descarboxilada; posteriormente, en las lagunas de resorción ósea, en donde predomina un $\mathrm{pH}$ ácido, la osteocalcina es carboxilada a su forma metabólicamente activa, la cual actúa sobre el osteoclasto, inhibiendo su diferenciación celular. De igual manera, la osteocalcina tiene un efecto directo sobre las células $\beta$ pancreáticas al aumentar la secreción de insulina y promover la sensibilidad periférica a la insulina ${ }^{(11,12)}$.

Estudios recientes, realizados en ratones alimentados con dieta alta en grasas, han demostrado que el hueso es un órgano que puede desarrollar resistencia a la insulina, esto conlleva disminución en la actividad de la osteocalcina y consecuentemente disminuye la sensibilidad de la insulina en adipocitos y miocitos ${ }^{(13-15)}$.

Los ácidos grasos saturados inducen ubiquitinación del receptor de insulina del osteoblasto, mediante un mecanismo que no se comparte con otras células clásicas sensibles a la insulina, lo que genera disminución en la señalización de la insulina en osteoblastos ${ }^{(13,16)}$.

Otro factor expresado por los osteoblastos es la osteopontina (OPN), al ser una glicoproteína de la matriz ex- tracelular tiene la capacidad de unirse a un gran número de receptores y funciona como mediadora en diferentes procesos biológicos. Tiene actividad sobre la mineralización ósea, que se debe a su capacidad de unirse al calcio y, por ende le permite tener una elevada afinidad por la hidroxiapatita. La OPN actúa en la mineralización ósea al tener una elevada afinidad por la hidroxiapatita, esto debido a su capacidad de unirse al calcio ${ }^{(17)}$.

Igualmente, la OPN puede actuar de manera similar a las citocinas, ya que participa como mediador inmune al interactuar con receptores de adhesión celular. Dichos receptores permiten que los osteoblastos pueden crear puentes de unión a la hidroxiapatita, lo cual va a permitir la conformación de una red integrada de comunicación entre las diversas células y la matriz extracelular ${ }^{(17,18)}$.

Por último, se ha descrito que uno de los efectos indirectos de la insulina es la disminución de la globulina ligadora de hormonas sexuales (SHBG), con el consecuente incremento de los niveles séricos de hormonas sexuales. Estudios clínicos realizados en mujeres con hiperinsulinemia mostraron un aumento de la producción de andrógenos en el ovario y reducción de la producción de SHBG en el hígado; este incremento de los niveles de andrógenos libres circulantes favorece el aumento de la $\mathrm{DMO}^{(19,20)}$ (figura 2).

Figura 2. Relación de insulina con la homeostasis del hueso y la glucosa

La insulina secretada por las células beta tiene acción sobre las células formadoras de hueso, las cuales secretan osteocalcina en su forma descarboxilada. Ésta va a aumentar la secreción de insulina en las células beta y va a aumentar la sensibilidad de la glucosa en diferentes tejidos. El osteoblasto también va secretar osteoprotegerina, la cual se va a unir al RANK-L, bloqueando y de esta manera inhibiendo la diferenciación del osteoclasto el cual en condiciones normales es estimulado por la osteocalcina, la cual se carboxila en las lagunas de reabsorción, gracias al pH bajo de estas.

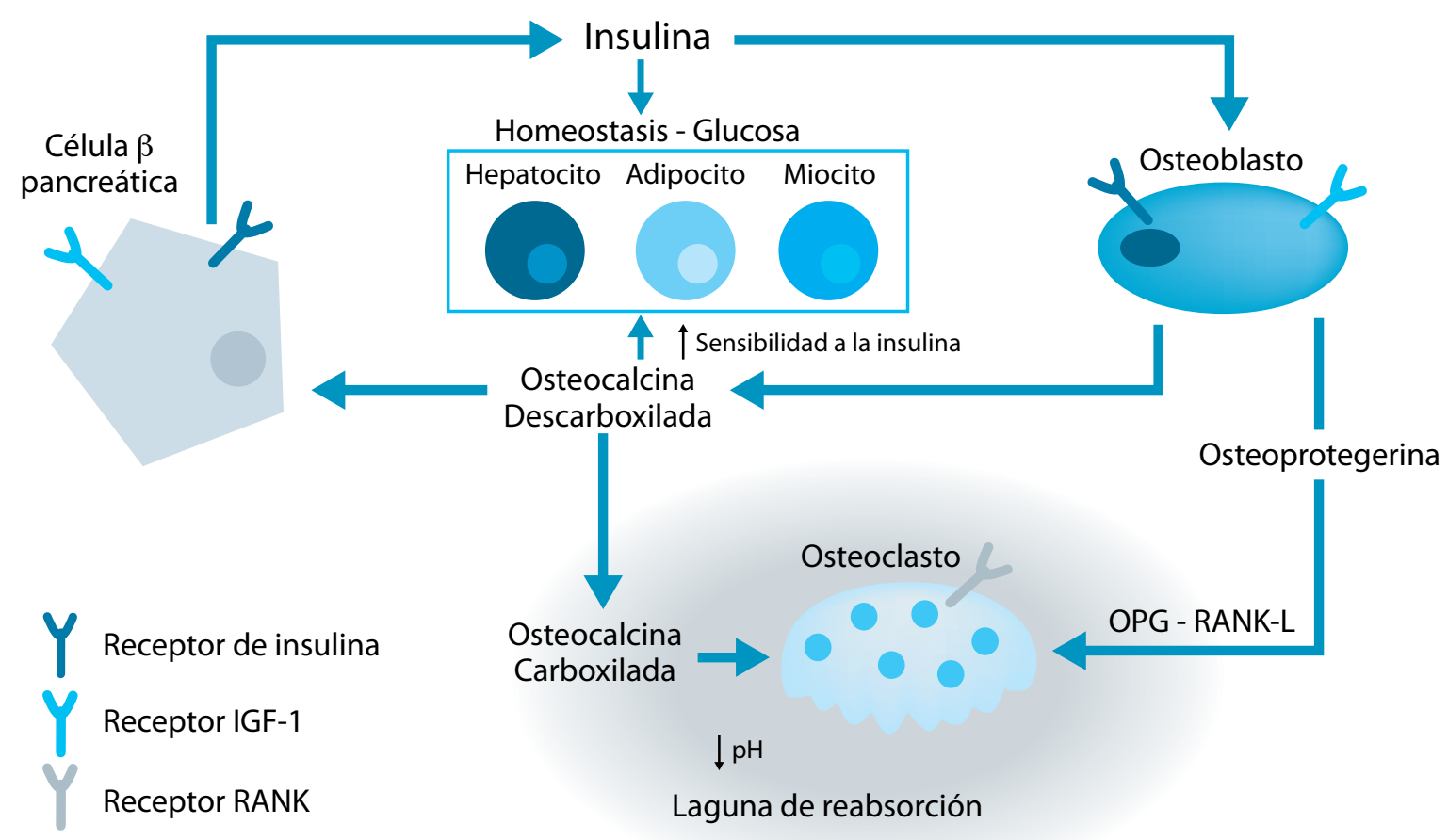




\section{IGF-1}

El factor de crecimiento similar a la insulina tipo 1 (IGF-1) se sintetiza en hepatocitos y osteoblastos; dicho factor actúa como señal que promueve la formación ósea y el crecimiento muscular ${ }^{(21,22)}$; además, está involucrado en el grosor, la longitud y la madurez del esqueleto. Su secreción está disminuida en estados de resistencia a la insulina como son la obesidad y el síndrome metabólico. En casos de deficiencia de IGF-1 ha sido reportada la presencia de baja DMO, disminución del tamaño del hueso y asociación con osteoporosis. Por lo anterior, se considera que la alteración del eje de IGF-1 en pacientes con obesidad o síndrome metabólico puede tener impacto en la densidad mineral ósea ${ }^{(22,23)}$.

\section{Vitamina D}

La vitamina D3 se sintetiza a partir del 7- dehidrocolesterol, que es escindido por rayos UVB a una longitud de onda de 270 a $300 \mathrm{~nm}$, con posteriores cambios conformacionales e hidroxilaciones, lo que promueve la síntesis de vitamina D metabólicamente $\operatorname{activa}^{(24)}$. La cantidad de vitamina D dependerá de la duración e intensidad de la exposición a los rayos UVB, que varían según la latitud y las estaciones climáticas. Se espera que bajo condiciones normales aproximadamente el $80 \%$ del total de la vitamina D sintetizada sea de dicho origen ${ }^{(25)}$.

Múltiples estudios han correlacionado de manera significativa bajos niveles séricos de $250 \mathrm{H}$-vitamina D con la prevalencia de obesidad, que sugieren un papel causal de direccionalidad incierta ${ }^{(26-28)}$. Entre las teorías propuestas acerca de dicha relación se encuentran: menor ingesta o absorción, menor disponibilidad de precursores de vitamina $\mathrm{D}$ en la piel e incluso aumento del catabolismo; sin embargo, no hay estudios que respalden alguna de estas teorías de manera consistente $^{(29) .}$

Se ha demostrado en adipocitos de ratones y humanos la presencia de la enzima CYP27B1 que se encarga de la conversión de $250 H$-vitamina D en $1,250 H$ vitamina D. Así mismo, esta última es inactivada por la enzima CYP24, también encontrada en tejido adiposo ${ }^{(30)}$. Por otro lado, existe evidencia de que la $1,250 H$ vitamina $\mathrm{D}$ inhibe la adipogénesis mediante la disminución de la expresión de genes como C/EBPb, PPARg y SREBP1, necesarios para la maduración de los adipocitos ${ }^{(30,31)}$.

De esta manera, se presenta un círculo vicioso en el que aparentemente la obesidad promueve un estado de hipovitaminosis D por secuestro en tejido adiposo. Dicha disminución de los niveles séricos de vitamina D perpetúa a su vez la adipogénesis mediante la pérdida de la inhibición en la maduración de los adipocitos. Un trabajo reciente encontró que la disminución de peso a dos años se relaciona con aumento en los niveles de $250 \mathrm{H}$ vitamina $\mathrm{D}$, lo que está a favor de un mecanismo de hipovitaminosis dependiente de almacenamiento en tejido $\operatorname{graso}^{(32)}$.

http://revistaendocrino.org/

\section{Adipocinas}

Leptina

La leptina es una hormona producida principalmente por los adipocitos, su función más conocida es la participación en la regulación del apetito. Los receptores de leptina se expresan en casi todos los tejidos, acentuando su alto pleiotropismo y su intervención en el gasto energético, reproducción, angiogénesis, inmunidad, curación de heridas y función cardiovascular, entre otras ${ }^{(33)}$.

El hueso tiene receptores sensibles a leptina en osteoblastos, osteoclastos y células de la médula ósea, lo que indica que esta hormona tiene un efecto directo sobre este órgano. A nivel periférico se ha demostrado que la leptina estimula la diferenciación de las células de la médula ósea a osteoblastos e inhibe su diferenciación a adipocitos ${ }^{(20)}$; también, la leptina regula directamente el desarrollo de los osteoclastos, reduciendo la producción de RANK y RANKL e incrementando la osteoprotegerina, lo que resulta en una inhibición de la osteoclastogénesis ${ }^{(34)}$.

En el hipotálamo existen receptores de leptina que generan efectos indirectos sobre el hueso. Centralmente, la leptina inhibe la producción y secreción de serotonina, permitiendo que el sistema nervioso simpático envié señales mediante la liberación de norepinefrina que es captada por los receptores $\beta 2$ adrenérgicos en los osteoblastos; esta señal produce supresión de la formación ósea e incrementa la resorción a través del aumento de la expresión de RANKL ${ }^{(35)}$.

La interacción de los efectos directos e indirectos de la leptina sobre el hueso tiene un resultado positivo en la densidad mineral ósea, siempre y cuando el balance sea predominante hacia el efecto periférico de la leptina sobre el hueso, sin embargo, si el efecto central de la leptina fuera dominante, habría una relación inversa.

En la interacción de los efectos directos e indirectos de la leptina sobre el hueso, el efecto periférico es predominante en la mayoría de circunstancias, esto sugiere que la integración final de las acciones de la leptina en el hueso es positiva ${ }^{(34)}$. Sin embargo, los pacientes obesos tienen niveles mucho más elevados de leptina, llegando a alcanzar cifras de 31,3 $\pm 24,1$ $\mathrm{ng} / \mathrm{ml}$ frente al rango que manejan los individuos con índice de masa corporal normal de 7,5 $\pm 9,3 \mathrm{ng} / \mathrm{ml}$. Numerosos estudios demuestran también que los pacientes hombres con IMC elevado presentan una mayor densidad mineral ósea; estos hallazgos refuerzan la correlación positiva existente entre leptina y densidad mineral ósea ${ }^{(35)}$.

\section{Adiponectina}

La adiponectina es una adipocina de 244 aminoácidos, tiene una estructura similar al colágeno VIII y X, y al factor de complemento $\mathrm{C} 1 \mathrm{q}^{(36)}$. Es secretada casi exclusivamente por adipocitos, principalmente del tejido adiposo visceral; sin embargo, la adiponectina está disminuida en la obesidad, ${ }^{(33)}$ 
encontrándose una correlación negativa con la obesidad en general y en particular con el tejido adiposo central( ${ }^{(37)}$. Su principal función consiste en regular la homeostasis de la glucosa; además, participa en el metabolismo lipídico y en los procesos antiinflamatorios $^{(36)}$. Cuenta con dos tipos de receptores (adipoR1 y adipoR2) que se expresan en diferentes tejidos, incluyendo el hueso, en el que se ha reportado expresión de estos receptores en osteoblastos y osteoclastos ${ }^{(38)}$.

Diferentes estudios sobre las acciones de la adiponectina no han logrado un consenso respecto a su papel en el metabolismo óseo. La adiponectina causa inhibición en la proliferación de los osteoblastos y favorece su apoptosis ${ }^{(34)}$. Asímismo, indirectamente aumenta la formación de osteoclastos mediante estimulación vía de RANKL e inhibición de osteoprotegerina en osteoblastos; la sumatoria de estos efectos deriva en disminución de la formación de hueso y genera disminución de la masa ósea ${ }^{(33,39)}$. Por otro lado, se ha demostrado que la adiponectina in vitro aumenta la proliferación de osteoblastos y su diferenciación, también causa la inhibición de la osteoclastogénesis ${ }^{(40)}$. Además, mediante un efecto central mediado por Fox01, la adiponectina inhibe la acción del sistema nervioso simpático, reduciendo el gasto energético y aumentando la formación ósea ${ }^{(41)}$.

\section{Hormonas sexuales}

\section{Estradiol}

El estradiol es una hormona sexual esteroidea, es considerado el principal estrógeno, se secreta principalmente por el ovario y por conversión periférica en el tejido adiposo a partir de precursores (andrógenos). Entre las múltiples funciones del estradiol se encuentra la participación en el eje gonadotropo, cierre de discos epifisiarios y mineralización ósea ${ }^{(42)}$. La concentración de esta hormona es mucho mayor en mujeres en edad reproductiva y presenta una caída abrupta posterior a la menopausia ${ }^{(43)}$.

El estradiol tiene efectos positivos sobre el hueso trabecular y cortical mediados por el receptor nuclear Er $\alpha$, que es expresado por osteoblastos y osteoclastos ${ }^{(44)}$. La estimulación de este receptor desencadena vías de señalización como el Wnt/ TCF, encargada de activar la expresión génica de componentes para la formación ósea; esto parece generar un efecto protector contra la resorción ósea en el tejido óseo endocortical, pero no en el hueso trabecular ${ }^{(45)}$.

La deficiencia de estradiol conlleva aumento en la diferenciación de osteoclastos y apoptosis acelerada de los osteocitos, manifestándose con un incremento en la resorción ósea, disminución de la DMO y aumento en el riesgo de sufrir fracturas $^{(45,47,48)}$. Se ha observado que el descenso de los niveles de estradiol se encuentra ampliamente relacionado con el riesgo de padecer osteoporosis, tanto en mujeres como en hombres ${ }^{(44)}$.

La aromatasa, que es la encargada de catalizar la conversión de andrógenos a estrógenos, se encuentra elevada en pa- cientes obesos, esto se debe al exceso de tejido adiposo blanco y factores proinflamatorios, como el TNF alfa, factores que tienen un efecto estimulatorio sobre la expresión génica de la $\operatorname{aromatasa}^{(49,50)}$.

Por consecuencia, los pacientes obesos presentan niveles elevados de estradiol, lo que se traduce en un incremento de la DMO y de la protección de la microarquitectura ósea ${ }^{(50,51)}$.

\section{Testosterona}

La testosterona es una hormona esteroidea perteneciente al grupo de los andrógenos. En los hombres esta hormona es secretada principalmente por las células testiculares de Leydig y es necesaria para la reproducción y función sexual ${ }^{(52,53)}$. Además, cumple funciones anabólicas que se han asociado con el incremento de la masa muscular y de la $\mathrm{DMO}^{(54,55)}$.

En el hueso, los receptores de andrógenos (AR) están presentes en células estromales de la médula ósea, osteoblastos, osteoclastos y osteocitos $^{(56)}$. En el proceso de mineralización ósea, la testosterona actúa de manera directa al reducir la resorción ósea mediante la inhibición de la diferenciación osteoclástica ${ }^{(7)}$. Igualmente, la testosterona estimula la producción de osteoprotegerina y la supresión de la expresión génica de RANKL en los osteoblastos, efectos que sumados inhiben indirectamente la formación de osteoclastos ${ }^{(55)}$.

Estudios previos han demostrado que individuos obesos presentan bajos niveles de testosterona sérica libre. Este efecto se ha asociado a un incremento en la expresión de la aromatasa $^{(57)}$. En individuos obesos se han encontrado niveles elevados de DMO, a pesar de los bajos niveles de testosterona, lo que indica que el efecto de esta hormona no es uno de los principales determinantes de la DMO, sugiriendo que dentro de las hormonas sexuales sobresale el estradiol como un mayor determinante de la $\mathrm{DMO}^{(58)}$.

\section{Conclusión}

Actualmente existe una gran controversia respecto a los efectos que ejerce la obesidad sobre el hueso, la mayoría de evidencia disponible apunta a disminución de riesgo de fractura en algunos sitios específicos, tales como fémur proximal, vértebras y cadera; sin embargo, existe mayor riesgo de fractura en húmero proximal (RR 1,28), y tobillo (RR 1,7)(59-61). Entre las teorías que podrían fundamentar la asociación positiva entre el mayor IMC y la DMO se encuentra la acción de la leptina y el aumento en la actividad de la aromatasa, esta última por incremento en la conversión periférica de estrógenos, determinantes claves para promover el proceso de formación ósea ${ }^{(62)}$. Por otra parte, recientemente se ha dilucidado una asociación entre la mayor cantidad de grasa y la DMO, encontrando que las citocinas proinflamatorias promueven la resorción ósea, lo que conlleva disminución en la $\mathrm{DMO}^{(63)}$. 
Existe una asociación positiva entre el IMC y la DMO, que se evidencia en la densitometría por absorción dual con rayos X (DXA); sin embargo, podría estar sujeta a errores de medición en algunos sitios donde existe mayor cantidad de tejidos blandos. No obstante, otros métodos cuantitativos de imagen (tomografía y ultrasonido) han denotado dicho incremento en la $\mathrm{DMO}^{(64-66)}$. Actualmente existen métodos más precisos y con alta resolución que permiten evaluar componentes como la microestructura y las distintas cargas en relación a la fuerza y consistencia ósea; un ejemplo es la tomografía cuantitativa periférica de alta precisión ( HR-pQCT) que ha demostrado mayor DMO en sujetos obesos comparados con sujetos con peso normal. A pesar de esto, se encuentran diferencias en cuanto a microestructura y mayores índices en la relación carga/ fuerza ósea en comparación con sujetos de peso normal ${ }^{(67,68)}$. Además, hay un incremento en la infiltración grasa a nivel muscular, lo que se asocia a pobre función muscular, riesgo de caídas y alto riesgo de fractura ${ }^{(69)}$.

En cuanto a las hormonas y factores endocrinos se encuentra la leptina, que es un reflejo de la cantidad de grasa corporal, involucrada en regulación del metabolismo energético y de la señalización del apetito a nivel hipotalámico ${ }^{(62)}$. Los efectos óseos de la leptina se deben a acción periférica donde directamente incrementa la formación ósea, y efectos indirectos desde nivel central, a través de receptores betaadrenérgicos del sistema nervioso autónomo, con impacto negativo sobre el hueso. Sin embargo, se sabe que predominan los efectos directos a nivel óseo y, por tanto, su acción es predominantemente $\operatorname{positiva}^{(19,70)}$.
La adiponectina es inversamente proporcional a la cantidad de masa grasa, tiene efectos con predominancia negativa en relación a la DMO, a través de la vía de señalización RANK/ RANK ligando/osteoprotegerina ${ }^{(8,71)}$. Estudios en ratones han documentado que podría regular el recambio óseo mediante la inervación autonómica, no obstante, dichos hallazgos no han sido reproducidos en humanos ${ }^{(72)}$.

En cuanto a las hormonas sexuales, el exceso de tejido adiposo promueve un incremento en la expresión de la aromatasa, quien incrementa la conversión periférica de andrógenos a estrógenos, lo que se traduce en un efecto predominantemente positivo en la formación ósea(73).

Los niveles séricos de vitamina D se encuentran bajos en la obesidad, debido al secuestro dado en el tejido adiposo, lo cual causa un mayor volumen de distribución. Sin embargo, no se ha dilucidado el impacto real de estos bajos niveles en los marcadores de salud ósea (DMO, recambio óseo) ni en el incremento en el riesgo de fractura ${ }^{(74)}$.

En conclusión, la evidencia actual sugiere que la obesidad se comporta como un factor protector contra algunas fracturas, principalmente fractura de cadera, y como un estimulante positivo en la formación ósea, dado por disminución en la resorción ósea e incremento en la fuerza ósea. Queda abierto el tema para la realización de nuevos estudios e investigación que permitan dilucidar a mayor profundidad la relación existente entre la obesidad y sus acciones en el hueso.

\section{Agradecimientos}

A la Universidad Nacional de Colombia

\section{Referencias}

1. WHO. WHO | Obesity and overweight. Who [Internet]. 2017 [cited 2017 Sep 3]; Available from: http://www.who.int/mediacentre/factsheets/fs311/ en/

2. Guh DP, Zhang W, Bansback N, Amarsi Z, Birmingham CL, Anis AH. The incidence of co-morbidities related to obesity and overweight: A systematic review and meta-analysis. BMC Public Health [Internet]. 2009 Dec 25 [cited 2017 Sep 3];9(1):88. Available from: http://bmcpublichealth.biomedcentral.com/articles/10.1186/1471-2458-9-88

3. Van Der Klaauw AA, Farooqi IS. The hunger genes: Pathways to obesity. Cell [Internet]. 2015 Mar 26 [cited 2017 Sep 1];161(1):119-32. Available from: http://www.ncbi.nlm.nih.gov/pubmed/25815990

4. Wang YC, McPherson K, Marsh T, Gortmaker SL, Brown M. Health and economic burden of the projected obesity trends in the USA and the UK. Lancet [Internet]. 2011 Aug 27 [cited 2017 Sep 1];378(9793):815-25. Available from: http://www.ncbi.nlm.nih.gov/pubmed/21872750

5. Greco EA, Lenzi A, Migliaccio S. The obesity of bone. Ther Adv Endocrinol Metab [Internet]. 2015 Dec 19 [cited 2017 Sep 3];6(6):273-86. Available from: http://journals.sagepub.com/doi/10.1177/2042018815611004

6. Rachner TD, Khosla S, Hofbauer LC. Osteoporosis: Now and the future. Lancet. 2011;377(9773):1276-87

7. Huber DM, Bendixen AC, Pathrose P, Srivastava S, Dienger KM, Shevde $\mathrm{NK}$, et al. Androgens suppress osteoclast formation induced by RANKL and macrophage- colony stimulating factor. Endocrinology [Internet]. 2001 Sep [cited 2017 Jun 26];142(9):3800-8. Available from: http:// www.ncbi.nlm.nih.gov/htbin-post/Entrez/query?db=m\&form=6\&dop $\mathrm{t}=\mathrm{r} \&$ uid $=11517156 \% 5 \mathrm{Cnhttp}: / /$ endo.endojournals.org/cgi/content $/$ full/142/9/3800\%5Cnhttp://endo.endojournals.org/cgi/content/abstract $/ 142 / 9 / 3800$
8. Luo XH, Guo LJ, Xie H, Yuan LQ, Wu XP, Zhou HD, et al. Adiponectin stimulates RANKL and inhibits OPG expression in human osteoblasts through the MAPK signaling pathway. J Bone Min Res [Internet]. 2006 Jul 17 [cited 2017 Sep 1];21(10):1648-56. Available from: http://www.ncbi.nlm.nih.gov/ pubmed/16995820

9. Vandewalle S, Taes Y, Van Helvoirt M, Debode P, Herregods N, Ernst C, et al. Bone size and bone strength are increased in obese male adolescents. J Clin Endocrinol Metab. 2013;98(7):3019-28.

10. Samuel VT, Shulman GI. Mechanisms for insulin resistance: Common threads and missing links. Cell [Internet]. 2012 Mar 2 [cited 2017 Sep 3];148(5):852-71. Available from: http://www.ncbi.nlm.nih.gov/ pubmed/22385956

11. Walsh JS. Normal bone physiology, remodelling and its hormonal regulation. Surg (United Kingdom) [Internet]. 2015 Jan 1 [cited 2017 Sep 3];33(1):1-6. Available from: http://linkinghub.elsevier.com/retrieve/pii/ S0263931914002257

12. Lecka-Czernik B, Stechschulte LA. Bone and fat: A relationship of different shades. Arch Biochem Biophys [Internet]. 2014 Nov [cited 2017 Sep 3];561:124-9. Available from: http://linkinghub.elsevier.com/retrieve/ pii/S0003986114002124

13. Wei J, Ferron M, Clarke CJ, Hannun YA, Jiang H, Blaner WS, et al. Bonespecific insulin resistance disrupts whole-body glucose homeostasis via decreased osteocalcin activation. J Clin Invest [Internet]. 2014 Apr 1 [cited 2017 Sep 3];124(4):1-13. Available from: https://www.jci.org/articles/ view/72323

14. Madeira E, Mafort TT, Madeira M, Guedes EP, Moreira RO, de Mendonça LMC, et al. Lean mass as a predictor of bone density and microarchitecture in adult obese individuals with metabolic syndrome. Bone [Internet]. 2014 
Feb [cited 2017 Sep 3];59:89-92. Available from: http://linkinghub.elsevier.com/retrieve/pii/S8756328213004407

15. Fulzele K, Riddle RC, DiGirolamo DJ, Cao X, Wan C, Chen D, et al. Insulin Receptor Signaling in Osteoblasts Regulates Postnatal Bone Acquisition and Body Composition. Cell [Internet]. 2010 [cited 2017 Sep 3];142(2):30919. Available from: http://www.sciencedirect.com/science/article/pii/ S0092867410006203

16. Ferron M, Wei J, Yoshizawa T, Del Fattore A, DePinho RA, Teti A, et al. Insulin Signaling in Osteoblasts Integrates Bone Remodeling and Energy Metabolism. Cell [Internet]. 2010 Jul 23 [cited 2017 Sep 3];142(2):296-308. Available from: http://www.ncbi.nlm.nih.gov/pubmed/20655470

17. O'Regan AW, Nau GJ, Chupp GL, Berman JS. Osteopontin (Eta-1) in cell-mediated immunity: teaching an old dog new tricks. ImmunolToday [Internet]. 2000 Oct [cited 2017 Jun 26];21(10):475-8. Available from: http://www. sciencedirect.com/science/article/pii/S0167569900017151

18. Naot D, Cornish J. Cytokines and hormones that contribute to the positive association between fat and bone. Front Endocrinol (Lausanne) [Internet]. 2014 [cited 2017 Sep 3];5(MAY):70. Available from: http://www.ncbi.nlm. nih.gov/pubmed/24847313

19. I.R. R. Fat and bone. Arch Biochem Biophys [Internet]. 2010 Nov 1 [cited 2017 Aug 30];503(1):20-7. Available from: http://ovidsp.ovid.com/ovidweb.cgi? $\mathrm{T}=J \mathrm{~S} \& \mathrm{PAGE}=$ reference\&D$=$ emed9\&NEWS $=\mathrm{N} \& A N=2010507817$

20. Sun AJ, Jing T, Heymsfield SB, Phillips GB. Relationship of leptin and sex hormones to bone mineral density in men. Acta Diabetol. 2003;40(SUPPL. 1).

21. Wong SK, Chin KY, Suhaimi FH, Ahmad F, Ima-Nirwana S. The relationship between metabolic syndrome and osteoporosis: A Review. Nutrients [Internet]. 2016 Jun 7 [cited 2017 Sep 1];8(6):347. Available from: http://www. ncbi.nlm.nih.gov/pubmed/27338453

22. Campos RMS, Lazaretti-Castro M, Mello MT De, Tock L, Silva PL, Corgosinho FC, et al. Influence of visceral and subcutaneous fat in bone mineral density of obese adolescents. Arq Bras Endocrinol Metabol [Internet]. 2012;56(1):12-8. Available from: http://www.ncbi.nlm.nih.gov/ pubmed/22460190

23. Aguirre GA, De Ita JR, de la Garza RG, Castilla-Cortazar I. Insulin-like growth factor-1 deficiency and metabolic syndrome. J Transl Med [Internet]. 2016 Jan 6 [cited 2017 Sep 1];14(1):3. Available from: http://www.translationalmedicine.com/content/14/1/3

24. Jensen MD, Ryan DH, Apovian CM, Ard JD, Comuzzie AG, Donato KA, et al. $2013 \mathrm{AHA} / \mathrm{ACC} / \mathrm{TOS}$ guideline for the management of overweight and obesity in adults: A report of the American college of cardiology/ American heart association task force on practice guidelines and the obesity society. J Am Coll Cardiol [Internet]. 2014 [cited 2017 Sep 1];63(25 PART B):2985-3023. Available from: http://dx.doi.org/10.1016/j. jacc. 2013.11.004\%5Cnpapers $3: / /$ publication/doi/10.1016/j. jacc.2013.11.004

25. Garvey W, Garber A, Mechanick J, Bray G, Dagogo-Jack S, Einhorn D, et al. American Association of Clinical Endocrinologists and American College of Endocrinology Position Statement on the 2014 Advanced Framework for a New Diagnosis of Obesity as a Chronic Disease. Endocr Pract [Internet]. 2014 [cited 2017 Sep 1];20(9):977-89. Available from: http://journals. aace.com/doi/abs/10.4158/EP14280.PS

26. Parikh SJ, Edelman M, Uwaifo GI, Freedman RJ, Semega-Janneh M, Reynolds J, et al. The Relationship between Obesity and Serum 1,25-Dihydroxy Vitamin D Concentrations in Healthy Adults. J Clin Endocrinol Metab [Internet]. 2004 Mar [cited 2017 Aug 30];89(3):1196-9. Available from: https://academic.oup.com/jcem/article-lookup/doi/10.1210/jc.2003-031398

27. Yao Y, Zhu L, He L, Duan Y, Liang W, Nie Z, et al. A meta-analysis of the relationship between vitamin D deficiency and obesity. Int J Clin Exp Med [Internet]. 2015 [cited 2017 Aug 30];8(9):14977-84. Available from: http:// www.ncbi.nlm.nih.gov/pubmed/26628980

28. Mallard SR, Howe AS, Houghton LA. Vitamin D status and weight loss: A systematic review and meta-analysis of randomized and nonrandomized controlled weight-loss trials. Am J Clin Nutr [Internet]. 2016 Oct 1 [cited 2017 Aug 30];104(4):1151-9. Available from: http://www.ncbi.nlm.nih. gov/pubmed/27604772

29. Vanlint S. Vitamin D and obesity. Nutrients [Internet]. 2013 Mar 20 [cited 2017 Aug 30];5(3):949-56. Available from: http://www.ncbi.nlm.nih.gov/ pubmed/23519290

30. Ding C, Gao D, Wilding J, Trayhurn P, Bing C. Vitamin D signalling in adipose tissue. Br J Nutr [Internet]. 2012 Dec 9 [cited 2017 Aug 30];108(11):191523. Available from: http://www.journals.cambridge.org/abstract_ S0007114512003285

31. Marcotorchino J, Tourniaire F, Landrier JF. Vitamin D, adipose tissue, and obesity. Horm Mol Biol Clin Investig [Internet]. 2013 Jan 1 [cited 2017 Aug 30];15(3):123-8. Available from: https://www.degruyter.com/view/j/ hmbci.2013.15.issue-3/hmbci-2013-0027/hmbci-2013-0027.xml

32. Ceglia L, Nelson J, Ware J, Alysandratos K-D, Bray GA, Garganta C, et al. Association between body weight and composition and plasma 25-hydroxyvitamin D level in the Diabetes Prevention Program. Eur J Nutr [Internet]. 2015 Feb 2 [cited 2017 Aug 30];56(1):1-10. Available from: http://www. ncbi.nlm.nih.gov/pubmed/26525562
33. Gómez-Ambrosi J, Rodríguez A, Catalán V, Frühbeck G. The bone-adipose axis in obesity and weight loss. Obes Surg [Internet]. 2008 Sep 19 [cited 2017 Jun 26];18(9):1134-43. Available from: http://www.ncbi.nlm.nih. gov/pubmed/18563500

34. Reid IR. Relationships between fat and bone. Osteoporos Int 2008;19(5):595-606.

35. Motyl KJ, Rosen CJ. Understanding leptin-dependent regulation of skeletal homeostasis. Biochimie [Internet]. 2012 Oct [cited 2017 Jun 26];94(10):2089-96. Available from: http://www.ncbi.nlm.nih.gov/ pubmed/22534195

36. Lago F, Dieguez C, Gómez-Reino J, Gualillo O. Adipokines as emerging mediators of immune response and inflammation. Nat Clin Pract Rheumatol [Internet]. 2007 Dec 1 [cited 2017 Sep 1];3(12):716-24. Available from: http://www.nature.com/doifinder/10.1038/ncprheum0674

37. Diamantis E, Troupis T, Farmaki P, Diamanti S, Skandalakis P. Obesity and fracture risk. Arch Hell Med [Internet]. 2016 [cited 2017 Sep 1];33(3):32030. Available from: http://www.ncbi.nlm.nih.gov/pubmed/25002873

38. Berner HS, Lyngstadaas SP, Spahr A, Monjo M, Thommesen L, Drevon CA et al. Adiponectin and its receptors are expressed in bone-forming cells. Bone [Internet]. 2004 Oct [cited 2017 Sep 1];35(4):842-9. Available from: http://www.ncbi.nlm.nih.gov/pubmed/15454091

39. Williams GA, Wang Y, Callon KE, Watson M, Lin JM, Lam JBB, et al. In vitro and in vivo effects of adiponectin on bone. Endocrinology [Internet]. 2009 Aug 1 [cited 2017 Sep 1];150(8):3603-10. Available from: https://academic.oup.com/endo/article-lookup/doi/10.1210/en.2008-1639

40. Oshima K, Nampei A, Matsuda M, Iwaki M, Fukuhara A, Hashimoto J, et al Adiponectin increases bone mass by suppressing osteoclast and activating osteoblast. Biochem Biophys Res Commun [Internet]. 2005 Jun 3 [cited 2017 Sep 1];331(2):520-6. Available from: http://www.ncbi.nlm.nih.gov/ pubmed/15850790

41. Dimitri P, Rosen C. The Central Nervous System and Bone Metabolism An Evolving Story. Calcif Tissue Int [Internet]. 2017 May 8 [cited 2017 Sep 1];100(5):476-85. Available from: http://www.ncbi.nlm.nih.gov/ pubmed/27501818

42. Pascual Serrano D, Vera Pasamontes C, Girón Moreno R. Modelos animales de dolor neuropático. Vol. 31, Dolor. 2016. 70-76 p.

43. David G, Gardner D, Dolores R. Greenspan's basic \& clinical endocrinology. McGraw-Hill, New York. McGraw-Hill Medical; 2011.

44. Henning P, Ohlsson C, Engdahl C, Farman H, Windahl SH, Carlsten H, et al The effect of estrogen on bone requires ERalpha in nonhematopoietic cells but is enhanced by ERalpha in hematopoietic cells. Am J Physiol Endocrinol Metab [Internet]. 2014 Oct 1 [cited 2017 Jun 26];307(7):E589-95. Available from: http://www.ncbi.nlm.nih.gov/pubmed/25117411

45. Bartell SM, Han L, Kim H, Kim SH, Katzenellenbogen J a, Katzenellenbogen BS, et al. Non-nuclear-initiated actions of the estrogen receptor protect cortical bone mass. Mol Endocrinol [Internet]. 2013 Apr [cited 2017 Jun 26];27(4):649-56. Available from: http://www.pubmedcentral.nih.gov/ articlerender.fcgi?artid=3607700\&tool=pmcentrez\&rendertype=abstract

46. Lim VW, Li J, Gong Y, Yuan JM, Wu TS, Hammond GL, et al. Serum free estradiol and estrogen receptor- $\alpha$ mediated activity are related to decreased incident hip fractures in older women. Bone [Internet]. 2012 Jun [cited 2017 Jun 26];50(6):1311-6. Available from: http://linkinghub.elsevier.com/retrieve/pii/S8756328212007247

47. Ho-Pham LT, Nguyen ND, Nguyen T V. Quantification of the relative contribution of estrogen to bone mineral density in men and women. BMC Musculoskelet Disord [Internet]. 2013 Dec 23 [cited 2017 Jun 26];14(1):366 Available from: http://www.pubmedcentral.nih.gov/articlerender.fcgi?arti $\mathrm{d}=3878025 \&$ tool $=$ pmcentrez\&rendertype $=$ abstract

48. Finigan J, Gossiel F, Glüer CC, Felsenberg D, Reid DM, Roux C, et al. Endogenous estradiol and the risk of incident fracture in postmenopausa women: The OPUS study. Calcif Tissue Int [Internet]. 2012 Jul 27 [cited 2017 Jun 26];91(1):59-68. Available from: http://www.ncbi.nlm.nih.gov/ pubmed/22644322

49. Bekaert M, Van Nieuwenhove Y, Calders P, Cuvelier CA, Batens AH, Kaufman JM, et al. Determinants of testosterone levels in human male obesity. Endocrine [Internet]. 2015 Sep 13 [cited 2017 Jun 26];50(1):202-11. Available from: http://www.ncbi.nlm.nih.gov/pubmed/25771885

50. Polari L, Yatkin E, Martínez Chacón MG, Ahotupa M, Smeds A, Strauss L, et al. Weight gain and inflammation regulate aromatase expression in male adipose tissue, as evidenced by reporter gene activity. Mol Cell Endocrinol [Internet]. 2015 Sep 5 [cited 2017 Jun 26];412:123-30. Available from http://www.ncbi.nlm.nih.gov/pubmed/26054748

51. Bredella MA, Lin E, Gerweck A V., Landa MG, Thomas BJ, Torriani M, et al. Determinants of bone microarchitecture and mechanical properties in obese men. J Clin Endocrinol Metab. 2012;97(11):4115-22.

52. Naderi S. Testosterone Replacement Therapy and the Cardiovascular System. Curr Atheroscler Rep [Internet]. 2016 Apr 1 [cited 2017 Jun 26];18(4):1-6. Available from: http://www.ncbi.nlm.nih.gov/pubmed/26932226

53. Mooradian AD, Morley JE, Korenman SG. Biological actions of androgens. Endocr Rev [Internet]. 1987 Feb [cited 2017 Jun 26];8(1):1-28. Available from: http://www.ncbi.nlm.nih.gov/pubmed/3549275 
54. Shin J, Sung J, Lee K, Song YM. Genetic influence on the association between bone mineral density and testosterone in Korean men. Osteoporos Int [Internet]. 2016 Feb 2 [cited 2017 Jun 26];27(2):643-51. Available from: http://www.ncbi.nlm.nih.gov/pubmed/26329099

55. Dabaja AA, Bryson CF, Schlegel PN, Paduch DA. The effect of hypogonadism and testosterone-enhancing therapy on alkaline phosphatase and bone mineral density. BJU Int [Internet]. 2015 Mar [cited 2017 Jun 26];115(3):480-5. Available from: http://www.ncbi.nlm.nih.gov/pubmed/25046796

56. Sinnesael M, Claessens F, Laurent M, Dubois V, Boonen S, Deboel L, et al. Androgen receptor (AR) in osteocytes is important for the maintenance of male skeletal integrity: Evidence from targeted AR disruption in mouse osteocytes. J Bone Miner Res [Internet]. 2012 Dec [cited 2017 Jun 26];27(12):2535-43. Available from: http://www.ncbi.nlm.nih.gov/ pubmed/22836391

57. Kawano $\mathrm{H}$, Sato $\mathrm{T}$, Yamada $\mathrm{T}$, Matsumoto $\mathrm{T}$, Sekine $\mathrm{K}$, Watanabe $\mathrm{T}$ et al. Suppressive function of androgen receptor in bone resorption. Proc Natl Acad Sci U S A [Internet]. 2003 Aug 5 [cited 2017 Jun 26];100(16):9416-21. Available from: http://www.ncbi.nlm.nih.gov/ pubmed/12872002\%5Cnhttp://www.pubmedcentral.nih.gov/articlerender.fcgi?artid=PMC170933\%5Cnhttp://www.pnas.org/content $/ 100 / 16 / 9416$.abstract?ijkey=81dcb86a407a154bb96210c0372da51 9b870d64e\&keytype2=tf_ipsecsha

58. Spoto B, Di Betta E, Mattace-Raso F, Sijbrands E, Vilardi A, Parlongo RM, et al. Pro- and anti-inflammatory cytokine gene expression in subcutaneous and visceral fat in severe obesity. Nutr Metab Cardiovasc Dis [Internet]. 2014 Oct [cited 2017 Jun 26];24(10):1137-43. Available from: http:// www.ncbi.nlm.nih.gov/pubmed/24984824

59. De Laet C, Kanis JA, Odén A, Johanson H, Johnell O, Delmas P, et al. Body mass index as a predictor of fracture risk: A meta-analysis. Osteoporos Int [Internet]. 2005 Nov 1 [cited 2017 Aug 30];16(11):1330-8. Available from: http://www.ncbi.nlm.nih.gov/pubmed/15928804

60. Compston JE, Watts NB, Chapurlat R, Cooper C, Boonen S, Greenspan S, et al. Obesity is not protective against fracture in postmenopausal women: Glow. Am J Med [Internet]. 2011 Nov [cited 2017 Aug 30];124(11):1043-50. Available from: http://www.ncbi.nlm.nih.gov/pubmed/22017783

61. Prieto-Alhambra D, Premaor MO, Fina Avilés F, Hermosilla E, Martinez-Laguna D, Carbonell-Abella C, et al. The association between fracture and obesity is site-dependent: A population-based study in postmenopausal women. J Bone Miner Res [Internet]. 2012 Feb [cited 2017 Aug 30];27(2):294-300. Available from: http://www.ncbi.nlm.nih.gov/pubmed/22095911

62. Cornish J, Callon KE, Bava U, Lin C, Naot D, Hill BL, et al. Leptin directly regulates bone cell function in vitro and reduces bone fragility in vivo. J Endocrinol [Internet]. 2002 Nov [cited 2017 Sep 1];175(2):405-15. Available from: http://www.ncbi.nlm.nih.gov/pubmed/12429038

63. Morley JE, Baumgartner RN. Cytokine-Related Aging Process. Journals Gerontol Ser A Biol Sci Med Sci [Internet]. 2004 Sep [cited 2017 Aug 30];59(9):M924-9. Available from: https://academic.oup.com/biomedgerontology/article-lookup/doi/10.1093/gerona/59.9.M924

64. Premaor MO, Comim FV, Compston JE. Obesity and fractures. Arq Bras Endocrinol Metabol [Internet]. 2014 Jul [cited 2017 Aug 30];58(5):470-7. Avail- able from:http://www.scielo.br/scielo.php?script=sci_arttext\&pid=S0004$27302014000500470 \& \operatorname{lng}=$ en\&nrm=iso\&tlng=en

65. Yang S, Shen X. Association and relative importance of multiple obesity measures with bone mineral density: the National Health and Nutrition Examination Survey 2005-2006. Arch Osteoporos [Internet]. 2015 Dec 9 [cited 2017 Aug 30];10(1):14. Available from: http://www.ncbi.nlm.nih. gov/pubmed/25957066

66. Knapp KM, Welsman JR, Hopkins SJ, Fogelman I, Blake GM. Obesity Increases Precision Errors in Dual-Energy X-Ray Absorptiometry Measurements. J Clin Densitom [Internet]. 2012 Jul [cited 2017 Aug 30];15(3):315-9. Available from: http://www.ncbi.nlm.nih.gov/pubmed/22402120\%0Ahttp:// linkinghub.elsevier.com/retrieve/pii/S1094695012000054

67. Evans AL, Paggiosi MA, Eastell R, Walsh JS. Bone density, microstructure and strength in obese and normal weight men and women in younger and older adulthood. J Bone Miner Res [Internet]. 2015 May [cited 2017 Aug 30];30(5):920-8. Available from: http://www.ncbi.nlm.nih.gov/ pubmed/25400253

68. Sornay-Rendu E, Boutroy S, Vilayphiou N, Claustrat B, Chapurlat RD. In obese postmenopausal women, bone microarchitecture and strength are not commensurate to greater body weight: The OS des femmes de Lyon (OFELY) study. J Bone Miner Res [Internet]. 2013 Jul [cited 2017 Aug 30];28(7):1679-87. Available from: http://www.ncbi.nlm.nih.gov/ pubmed/23371055

69. Himes CL, Reynolds SL. Effect of obesity on falls, injury, and disability. J Am Geriatr Soc [Internet]. 2012 Jan [cited 2017 Aug 30];60(1):124-9. Available from: http://www.ncbi.nlm.nih.gov/pubmed/22150343

70. Hamrick MW, Ferrari SL. Leptin and the sympathetic connection of fat to bone. Osteoporos Int [Internet]. 2008 Jul [cited 2017 Aug 30];19(7):90512. Available from: http://www.ncbi.nlm.nih.gov/pubmed/17924050

71. Lenchik L, Register TC, Hsu FC, Lohman K, Nicklas BJ, Freedman BI, et al. Adiponectin as a novel determinant of bone mineral density and visceral fat. Bone [Internet]. 2003 Oct [cited 2017 Aug 30];33(4):646-51. Available from: http://linkinghub.elsevier.com/retrieve/pii/S8756328203002370

72. Kajimura D, Lee HW, Riley KJ, Arteaga-Solis E, Ferron M, Zhou B, et al. Adiponectin regulates bone mass via opposite central and peripheral mechanisms through foxo1. Cell Metab [Internet]. 2013 Jun 4 [cited 2017 Aug 30];17(6):901-15. Available from: http://www.ncbi.nlm.nih.gov/ pubmed/23684624

73. Riis BJ, Rødbro P, Christiansen C. The role of serum concentrations of sex steroids and bone turnover in the development and occurrence of postmenopausal osteoporosis. Calcif Tissue Int [Internet]. 1986 Jun [cited 2017 Sep 1];38(6):318-22. Available from: http://www.ncbi.nlm.nih.gov/ pubmed/3089552

74. Walsh JS, Evans AL, Bowles S, Naylor KE, Jones KS, Schoenmakers I, et al. Free 25-hydroxyvitamin $\mathrm{D}$ is low in obesity, but there are no adverse as sociations with bone health. Am J Clin Nutr [Internet]. 2016 Jun 1 [cited 2017 Aug 30];103(6):1465-71. Available from: http://www.ncbi.nlm.nih. gov/pubmed/27169839. 